\title{
Elevated resting heart rates are a risk factor for mortality among patients with coronavirus disease 2019 in Wuhan, China
}

\author{
Han Jin ${ }^{1 \#}$, Shengwen Yang ${ }^{2 \#}$, Fan Yang ${ }^{1 \#}$, Long Zhang ${ }^{1 \#}$, Haoyu Weng ${ }^{1 \#}$, Shengcong \\ Liu $^{1 \#}$, Fangfang Fan ${ }^{1}$, Haichao $\mathrm{Li}^{3}$, Xizi Zheng ${ }^{4}$, Hongyu Yang ${ }^{4}$, \\ Yan Zhang ${ }^{1}$, Jing Zhou ${ }^{1}$, Jianping $\mathbf{L i}^{1}$ \\ 'Department of Cardiology, Peking University First Hospital, Beijing 100034, China; \\ ${ }^{2}$ The Department of Cardiology, Affiliated Beijing Chaoyang Hospital of Capital Medical University, Beijing, \\ 100020, China; \\ ${ }^{3}$ Department of Pulmonary Medicine, Peking University First Hospital, Beijing 100034, China; \\ ${ }^{4}$ Department of Nephrology, Peking University First Hospital, Beijing 100034, China
}

\#These authors contributed equally to this work.

Address for Correspondence: Dr. Jianping Li, Department of Cardiology, Peking University First Hospital, No. 8 Xishiku St, Xicheng District, Beijing 100034, China.

Email: lijianping03455@pkufh.com

Dr. Jing Zhou, Department of Cardiology, Peking University First Hospital, No. 8 Xishiku St, Xicheng District, Beijing 100034, China

Email: zhoujing1232004@sina.com

\begin{tabular}{|l|}
\hline Access this article online \\
\hline $\begin{array}{l}\text { Website: } \\
\text { www.intern-med.com }\end{array}$ \\
\hline DOI: \\
10.2478/jtim-2021-0042 \\
\hline Quick Response Code: \\
\hline \\
\hline \\
\hline
\end{tabular}

\section{ABSTRACT}

Background: We evaluated the association between higher resting heart rates (RHRs) and adverse events in COVID-19 patients. Methods: One hundred and thirty-six patients with laboratory-confirmed COVID-19 were admitted. Outcomes of patients with different RHRs were compared. Results: Twenty-nine patients had RHRs of $<80$ bpm (beat per min), 85 had $80-99$ bpm and 22 had $\geq 100$ bpm as tachycardia. Those with higher RHRs had lower pulse oxygen saturation $\left(\mathrm{SpO}_{2}\right)$ and higher temperatures, and there was a higher proportion of men upon admission (all $P<0.05$ ). Patients with higher RHRs showed higher white blood cell counts and D-dimer, cardiac troponin I ( Tnl), N-terminal pro-B-type natriuretic peptide and hypersensitive $C$-reactive protein levels, but lower albumin levels (all $P<0.05)$ after admission. During follow-up, 26 patients died (mortality rate, 19.1\%). The mortality rate was significantly higher among patients with tachycardia than among the moderate and low RHR groups (all $P<0.001)$. Kaplan-Meier survival curves showed that the risks of death and ventilation use increased for patients with tachycardia $(P<0.001)$. Elevated RHR as a continuous variable and a mean RHR as tachycardia were independent risk factors for mortality and ventilator use (all $P<0.05$ ) in the multivariable adjusted Cox proportional hazards regression model. Conclusions: Elevated average RHRs during the first 3 days of hospitalisation were associated with adverse outcomes in COVID-19 patients. Average RHRs as tachycardia can independently predict all-cause mortality.

Key words: coronavirus disease 2019 , resting heart rate, tachycardia, risk factor, prognosis

\section{INTRODUCTION}

Since December 2019, coronavirus disease 2019 (COVID-19), caused by severe acute respiratory syndrome coronavirus 2 (SARSCoV-2) infection, has fully erupted and become prevalent in over 200 countries worldwide. On 16 April 2021, the World Health Organization reported that over 137 million cases of COVID-19 have been confirmed in various countries, with over 2 million deaths. ${ }^{[1]}$ Among the COVID-19 infections, individual clinical manifestations vary greatly. As the disease first emerged in Wuhan, China, after patients' admission to our hospital, we intensively monitored their vital signs, including heart rate, blood pressure and body temperature, for three consecutive days. If their symptoms improved or stabilised, the monitoring was reduced to once daily owing to restricted conditions and lack of manpower. During the first 3 days of intensive vital sign monitoring, patients with different resting 
heart rate (RHR) levels were observed. At present, the association between specific vital signs during COVID-19 infection and prognosis remains unknown. Previous studies have shown that significant heart rate changes are often associated with poor outcomes in most diseases. ${ }^{[2-4]}$ The present study, therefore, retrospectively analysed the heart rate data for COVID-19 patients in the early stages of admission to determine a correlation between heart rate changes and clinical prognosis to help enrich and improve clinical treatment and prognosis evaluations of COVID-19 patients.

\section{METHODS}

\section{Study participants}

This single-centre, retrospective cohort study included 136 COVID-19 patients in the ward of the Zhongfaxincheng campus of Tongji Hospital, Tongji Medical College, Huazhong University of Science \& Technology (Wuhan, China) who were cared for by the Peking University Medical Team beginning 9 February 2020 (the last patient in our study was admitted on 20 February and the endpoints within 30 days were observed in all patients till 20 March). All patients were confirmed to be infected with SARSCoV-2 in secondary or Fangcang hospitals and transported to our ward due to aggravation of the disease and critical care demands. The diagnosis was mainly based on the positive of the nuclear acid, the Viral Nucleic Acid Kit (Health) was used to extract nucleic acids from the clinical samples according to the kit instructions. Patients were diagnosed according to the World Health Organization's interim guidance. ${ }^{[5]}$ All definitions we used were for adults. The Ethics Committee of Peking University First Hospital approved this study (the ethics approval number is 2020-keyan-135), and all procedures and researches were in accordance with the 'Declaration of Helsinki'. ${ }^{[6]}$

\section{Patient clinical data collection}

General clinical data of the enrolled patients were collected from the electronic medical record system and included demographics (age and sex), clinical data (signs, symptoms, chronic medical illnesses, treatment and clinical outcomes) and laboratory findings. Owing to restricted conditions, the RHRs were collected at 6:00, 10:00, 14:00 and 20:00 hours using finger oxygen monitoring equipment with patients resting in recumbency. The RHRs were then averaged.

Laboratory testing included the highest serum $\mathrm{N}$-terminal pro-B-type natriuretic peptide (NT-proBNP), cardiac troponin I (TnI), hypersensitive C-reactive protein (hsCRP) and blood creatinine values as well as the lowest haemoglobin $(\mathrm{Hb})$ value. The normal reference range for TnI uses an upper limit of $\leq 26.3 \mathrm{ng} / \mathrm{L}$, which is the $99 \mathrm{th}$ percentile for healthy people.
All patients were treated as per the 'Diagnosis and Treatment Protocol for Novel Coronavirus Pneumonia (Trial Version 5-7) ${ }^{.}{ }^{[7-9]}$ The clinical outcomes (all-cause mortality and invasive and non-invasive ventilator use) were monitored. Discharge criteria are as follows: 1) body temperature is back to normal for more than 3 days; 2) respiratory symptoms show obvious improvement; 3) pulmonary imaging shows obvious absorption of inflammation and 4) nucleic acid tests negative twice consecutively on respiratory tract samples such as sputum and nasopharyngeal swabs (sampling interval being at least $24 \mathrm{~h}$ ). Those who meet all the criteria can be discharged.

\section{Definitions}

Fever was defined as an axillary temperature of $\geq 37.3^{\circ} \mathrm{C}$. Upward-trending RHRs were defined as RHRs showing a straight upward trend for 3 days or average RHRs on days 2 and 3 exceeding that on day 1 after admission. Downward-trending RHRs were defined as RHRs showing a straight downward trend for 3 days or average RHRs on days 2 and 3 being less than that on day 1 after admission. We calculated the change in RHRs ( $\triangle$ RHR) by subtracting the average RHR on day 1 from the average RHRs on days 2 and 3 . These changes in RHRs reflect upward and downward trends.

\section{Statistical analysis}

All analyses were conducted using SPSS, version 22.0 (IBM Corp, Armonk, NY, USA) for Windows and R software version 3.3.2 (http://www.R-project.org). Continuous variables are expressed as the median (interquartile range $[\mathrm{QR}])$ and categorical variables as a number or percentage. Patient groups were compared using the Kruskal-Wallis rank test for continuous variables and the chi-square or Fisher's exact tests for categorical variables as appropriate. Cox regression analysis was used to determine response rate-associated predictors. Univariate analysis was conducted, followed by multivariate analysis adjusting for the factors, so that the univariate analyses were $P<0.05$. The variables were selected as follows. Sex and age were forced into adjusted model I. Pulse oxygen saturation $\left(\mathrm{SpO}_{2}\right)$, average temperature for 3 days, NT-proBNP, TnI, hs-CRP, D-dimer, albumin and white blood cells (WBC) count values were selected for entry in model II on the basis of $P<0.05$ according to the baseline. History of coronary artery disease, $\beta$-blocker use and creatinine values were forced into the models for useful clinical meanings. Survival curves were plotted using Kaplan-Meier curves and compared between patients in the different RHR categories. Because almost all death events occurred within 1 month after treatment, we only calculated 30-day mortality and considered the incidents occurring within 30 days of admission. All Kaplan-Meier curves and Cox regression analyses were based on a 30-day observation. 


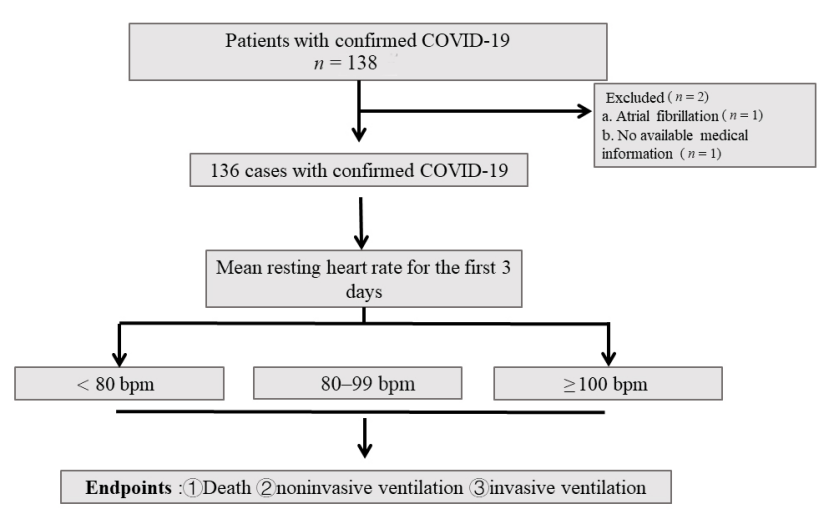

Figure 1: Flowchart of patient recruitment. COVID-19: coronavirus disease 2019; bpm: beat per min.

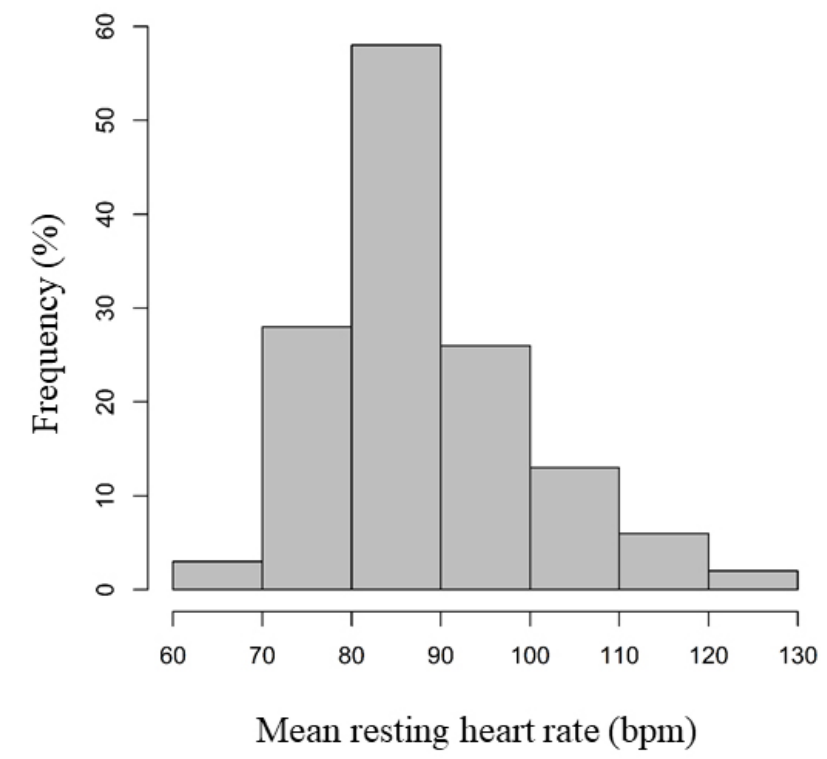

Figure 2: Histogram of mean resting heart rate for the first 3 days after admission (divided into 10-bpm intervals)

Adjusted models (the same variables adjusted in model II) were constructed using a restricted cubic spline with four knots to flexibly display the relationship between the hazard of developing an outcome and the continuous covariate of mean RHRs and $\triangle$ RHR. $P<0.05$ was considered statistically significant. All reported $P$-values are two-tailed.

\section{RESULTS}

Figure 1 shows the flowchart for patient recruitment. One hundred and thirty-eight patients with confirmed COVID-19 infections were admitted from 6 February 2020 to 20 March 2020. One patient with an atrial fibrillation history and one patient with missing laboratory results were excluded (the patient died on the day after admission); thus, 136 patients were enrolled. The groups were divided according to the mean RHRs for the first 3 days, with 29 patients $(21.3 \%$ ) having RHRs of $<80 \mathrm{bpm}$ (beat per min), 85 patients $(62.5 \%)$ having RHRs of $80-99 \mathrm{bpm}$ and 22 patients $(16.2 \%)$ having RHRs of $\geq 100 \mathrm{bpm}$, defined as tachycardia. Figure 2 shows the RHR distribution.

Table 1 presents the patients' demographic and clinical characteristics. The median age was 66 years (IQR 54-72 years); 70 patients $(51.5 \%)$ were men and $30(22.1 \%)$ had smoking histories. Fever (117 [86.0\%]) was the most common symptom, followed by coughing (114 [83.8\%]), shortness of breath (99 [66.2\%]), fatigue (84 [61.8\%]) and sputum production $(84[61.8 \%])$. Sixty-two patients $(45.6 \%)$ had hypertension, the most common comorbidity. Twentysix (19.1\%), 21 (15.4\%), 9 (6.6\%) and $27(19.9 \%)$ patients had coronary artery disease, chronic pulmonary disease, chronic renal disease and diabetes, respectively (Table 1). Compared with patients in the lower and moderate mean RHR groups, those with higher RHRs had lower $\mathrm{SpO}_{2}$ (median [range]: 97\% [92-98] vs. 95\% [92-97] vs. 86\% [73-94]; $P<0.001)$ and a higher proportion of men $(14$ [48.3\%] vs. $39[45.9 \%]$ vs. $17[77.3 \%] ; P=0.030)$ upon admission (Table 1).

Patients with higher mean RHRs showed higher median WBC counts and D-dimer, TnI, NT-proBNP and Hs-CRP levels, but lower median albumin levels post-admission, with significant differences found in all cases (all $P<0.05$; Table 1).

Before admission, 116 of 136 patients (85.3\%) had taken antiviral drugs (most patients had taken one from arbidol, oseltamivir, ribavirin and lopinavir/ritonavir) and 63 patients $(46.3 \%)$ had taken antibiotic treatment. Twentyfive patients $(18.4 \%)$ had been given prescriptions for intravenous immunoglobulin therapy, 18 patients (13.2\%) for non-steroidal anti-inflammatory drugs (NSAIDs) and 32 patients (23.5\%) for glucocorticoids. Patients with higher mean RHRs showed higher proportion of antibiotic treatment and glucocorticoid prescription (all $P<0.05$; Table 1).

During the 30-day observation, 31 of 136 patients $(22.8 \%)$ were treated with non-invasive ventilation and 12 of 136 $(8.8 \%)$ were treated with invasive mechanical ventilation. A total of 63 patients $(46.3 \%)$ were discharged; the percentage of patients discharged from the hospital was significantly higher among patients with lower mean RHR compared to patients with higher RHR $(62.1 \%$ vs. $51.7 \%$ vs. $4.5 \%$; $P$ $=0.002 ;$ Table 1). Twenty-six patients (19.1\%) died, and the mortality rate was significantly higher in patients with higher RHRs than in the moderate and low RHR groups (63.6\% vs. $12.9 \%$ vs. $3.4 \%$, respectively; $P<0.001$; Table 1 ).

Kaplan-Meier survival curves (Figures 3-5) showed that 
Jin et al.: RHRs in COVID-19

\begin{tabular}{|c|c|c|c|c|c|}
\hline Variable & All & Group $1<80 \mathrm{bpm}$ & Group 2 80-99 bpm & Group $3 \geq 100$ bpm & $P$-value \\
\hline No. of patients & 136 & 29 & 85 & 22 & \\
\hline Age, median(range), years & $66(54-72)$ & $67(57-78)$ & $65(52-70)$ & $65(52-74)$ & 0.152 \\
\hline Male, \% & $70(51.5 \%)$ & $14(48.3 \%)$ & $39(45.9 \%)$ & $17(77.3 \%)^{a, b}$ & 0.030 \\
\hline Smoking, \% & $30(22.1 \%)$ & $8(27.6 \%)$ & $14(16.5 \%)$ & $8(36.4 \%)$ & 0.604 \\
\hline \multicolumn{6}{|l|}{ Signs and symptoms } \\
\hline $\mathrm{SpO}_{2}$ at admission, $\%$ & $95(89-97)$ & $97(92-98)$ & $95(92-97)$ & $86(73-94)^{a, b}$ & $<0.001$ \\
\hline $\mathrm{SBP}$ at admission, $\mathrm{mmHg}$ & $134(117-149)$ & $136(120-163)$ & $132(115-148)$ & $92(125-156)$ & 0.196 \\
\hline DBP at admission, $\mathrm{mmHg}$ & $83(72-94)$ & $85(74-98)$ & $82(73-91)$ & $94(69-98)$ & 0.543 \\
\hline Temperature for 3 days, ${ }^{\circ} \mathrm{C}$ & $36.6(36.4-36.9)$ & $36.6(36.4-36.8)$ & $36.6(36.4-36.9)$ & $36.7(36.5-36.9)$ & 0.062 \\
\hline Fever, \% & $117(86.0 \%)$ & $23(79.3 \%)$ & $74(87.1 \%)$ & $20(90.9 \%)$ & 0.651 \\
\hline Cough, \% & $114(83.8 \%)$ & $22(75.9 \%)$ & $73(85.9 \%)$ & $19(86.4 \%)$ & 0.422 \\
\hline Sputum production, \% & $84(61.8 \%)$ & $17(60.7 \%)$ & $51(60.0 \%)$ & $16(72.7 \%)$ & 0.538 \\
\hline Shortness of breath, \% & $99(66.2 \%)$ & $18(62.1 \%)$ & $58(68.2 \%)$ & $14(63.6 \%)$ & 0.801 \\
\hline Chest pain, \% & $25(18.4 \%)$ & $5(17.2 \%)$ & $17(20.0 \%)$ & $3(183.6 \%)$ & 0.777 \\
\hline Sore throat, \% & $29(21.3 \%)$ & $7(24.1 \%)$ & $20(23.5 \%)$ & $2(9.1 \%)$ & 0.309 \\
\hline Diarrhoea, \% & $70(51.5 \%)$ & $18(62.1 \%)$ & $42(49.4 \%)$ & $10(45.5 \%)$ & 0.413 \\
\hline Nausea, \% & $46(33.8 \%)$ & $13(44.8 \%)$ & $28(32.9 \%)$ & $5(22.7 \%)$ & 0.246 \\
\hline Vomiting, \% & $28(20.6 \%)$ & $7(24.1 \%)$ & $16(18.8 \%)$ & $5(22.7 \%)$ & 0.800 \\
\hline Stomach ache, \% & $25(18.4 \%)$ & $8(27.6 \%)$ & $14(16.5 \%)$ & $3(13.6 \%)$ & 0.337 \\
\hline Headache, \% & $47(34.6 \%)$ & $10(34.5 \%)$ & $29(34.1 \%)$ & $8(35.4 \%)$ & 0.981 \\
\hline Muscle ache, \% & $64(47.1 \%)$ & $14(48.3 \%)$ & $39(46.4 \%)$ & $11(50.0 \%)$ & 0.951 \\
\hline Fatigue, $\%$ & $84(61.8 \%)$ & $20(69.0 \%)$ & $51(60.7 \%)$ & $13(59.1 \%)$ & 0.693 \\
\hline \multicolumn{6}{|l|}{$\begin{array}{l}\text { Chronic medical illness } \\
\text { and treatment }\end{array}$} \\
\hline Coronary artery disease, \% & $26(19.1 \%)$ & $9(31.0 \%)$ & $15(17.6 \%)$ & $2(9.1 \%)$ & 0.122 \\
\hline Hypertension, \% & $62(45.6 \%)$ & $15(51.7 \%)$ & $40(47.1 \%)$ & $7(31.8 \%)$ & 0.334 \\
\hline $\begin{array}{l}\text { Chronic pulmonary disease, } \\
\%\end{array}$ & $21(15.4 \%)$ & $5(17.2 \%)$ & $11(12.9 \%)$ & $5(22.7 \%)$ & 0.503 \\
\hline Chronic renal disease, \% & $9(6.6 \%)$ & $2(6.9 \%)$ & $4(4.7 \%)$ & $3(13.6 \%)$ & 0.323 \\
\hline Diabetes, \% & $27(19.9 \%)$ & $5(17.2 \%)$ & $18(21.2 \%)$ & $4(18.2 \%)$ & 0.880 \\
\hline$\beta$-blocker, \% & $13(9.6 \%)$ & $4(13.8 \%)$ & $9(10.6 \%)$ & $0(0 \%)$ & 0.220 \\
\hline \multicolumn{6}{|l|}{ Laboratory test (IQR) } \\
\hline WBC, $\times 10^{9}$ per $\mathrm{L}$ & $5.52(4.38-7.75)$ & $5.50(4.83-7.64)$ & $5.05(4.27-6.36)$ & $11.8(7.54-13.42)^{a, b}$ & $<0.001$ \\
\hline Haemoglobin, g/L & $124(115-138)$ & $122(113-129)$ & $123(115-137)$ & $140(122-153)$ & 0.240 \\
\hline Albumin, g/L & $34.3(30.7-91.0)$ & $33.5(31.0-37.3)$ & $35.1(31.4-38.5)$ & $31.3(29.0-35.1)^{b}$ & 0.016 \\
\hline Creatinine, $\mu \mathrm{mol} / \mathrm{L}$ & $73.5(58.0-91.0)$ & $71.0(57.5-90.5)$ & $71.0(57.0-89.5)$ & $83.5(63.3-100.5)$ & 0.434 \\
\hline D-dimer, $\mu \mathrm{g} / \mathrm{mL}$ & $1.26(0.52-2.52)$ & $0.83(0.46-1.92)$ & $1.14(0.48-2.03)$ & $2.62(1.57-2.61)^{a, b}$ & $<0.001$ \\
\hline $\mathrm{Tnl}, \mathrm{pg} / \mathrm{mL}$ & $4.7(2.2-10.3)$ & $5.0(2.3-10.6)$ & $4.0(1.9-7.3)$ & $28.7(3.7-154.8)^{\mathrm{a}, \mathrm{b}}$ & 0.007 \\
\hline NT-proBNP, pg/mL & $180.0(67.0-460.7)$ & $284.0(116.0-783.0)$ & $138.50(62.5-264.3)$ & $852.0(226.5-1551.5)^{a, b}$ & 0.047 \\
\hline $\mathrm{Hs}-\mathrm{CRP}, \mathrm{mg} / \mathrm{L}$ & $35.7(6.2-82.5)$ & $19.9(5.4-59.7)$ & $27.5(5.1-68.3)$ & 106.9(37.6-212.9) & $<0.001$ \\
\hline \multicolumn{6}{|l|}{ Treatment } \\
\hline Antiviral treatment, \% & $116(85.3 \%)$ & $25(86.2 \%)$ & $75(88.2 \%)$ & $16(72.7 \%)$ & 0.185 \\
\hline Antibiotic treatment, \% & $63(46.3 \%)$ & $12(41.4 \%)$ & $33(38.8 \%)$ & $18(81.8 \%)^{\mathrm{a}, \mathrm{b}}$ & 0.001 \\
\hline $\begin{array}{l}\text { Intravenous immunoglobulin } \\
\text { therapy, \% }\end{array}$ & $25(18.4 \%)$ & $5(17.2 \%)$ & $13(15.3 \%)$ & $7(31.8 \%)$ & 0.201 \\
\hline NSAIDs, $\%$ & $18(13.2 \%)$ & $2(6.9 \%)$ & $15(17.6 \%)$ & $1(4.5 \%)$ & 0.142 \\
\hline Glucocorticoids, \% & $32(23.5 \%)$ & $3(10.3 \%)$ & $15(17.6 \%)$ & $14(63.6 \%)^{a, b}$ & $<0.001$ \\
\hline Non-invasive ventilation, $\%$ & $31(22.8 \%)$ & $1(3.4 \%)$ & $14(16.5 \%)$ & $16(72.7 \%)^{a, b}$ & $<0.001$ \\
\hline $\begin{array}{l}\text { Invasive mechanical } \\
\text { ventilation, \% }\end{array}$ & $12(8.8 \%)$ & $1(3.4 \%)$ & $2(2.4 \%)$ & $9(40.9 \%)^{a, b}$ & $<0.001$ \\
\hline \multicolumn{6}{|l|}{ Clinical outcomes } \\
\hline Remained in hospital, \% & $47(34.6 \%)$ & $10(34.5 \%)$ & $32(35.3 \%)$ & $7(31.8 \%)$ & 0.954 \\
\hline Discharged, \% & $63(46.3 \%)$ & $18(62.1 \%)$ & $44(51.7 \%)$ & $1(4.5 \%)^{a, b}$ & 0.002 \\
\hline Died, \% & $26(19.1 \%)$ & $1(3.4 \%)$ & $11(12.9 \%)$ & $14(63.6 \%)^{a, b}$ & $<0.001$ \\
\hline
\end{tabular}

aCompared with lower RHR, $P<0.05$; ${ }^{\text {b}}$ compared with moderate $\mathrm{RHR}, P<0.05$.

IQR: interquartile range; RHR: resting heart rate; $\mathrm{SpO}_{2}$ : pulse oxygen saturation; SBP: systolic blood pressure; DBP: diastolic blood pressure; WBC:

white blood cells; Tnl: troponin I; CK-MB: creatinine kinase MB isoenzyme; NT-proBNP: N-terminal pro-B-type natriuretic peptide; Hs-CRP: hypersensitive

C-reactive protein; NSAIDs: non-steroidal anti-inflammatory drugs; bpm: beat per min. 


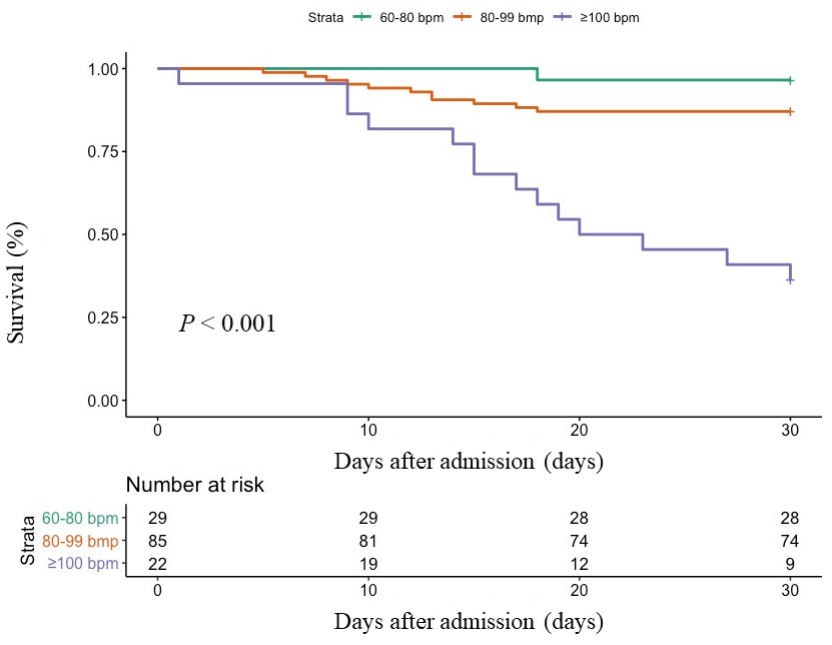

Figure 3: Kaplan-Meier estimates of mortality by mean resting heart rate

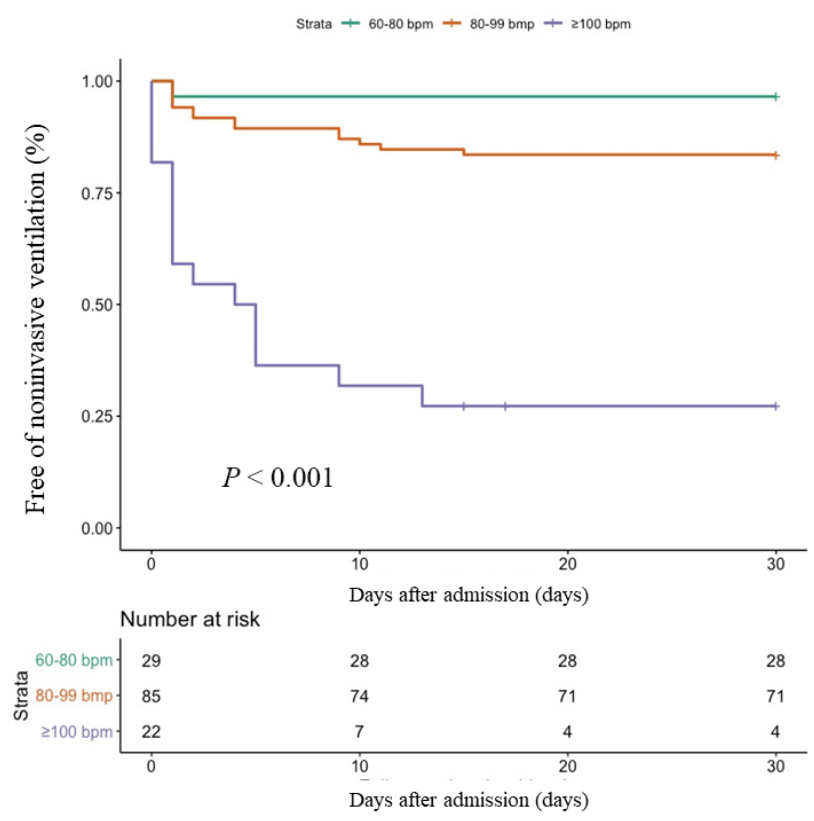

Figure 4: Kaplan-Meier estimates of patients not requiring non-invasive ventilation use by mean resting heart rate. bpm: beat per min.

the risks of death, non-invasive ventilation and invasive mechanical ventilation were increased in patients with tachycardia (all $P<0.001$ ). The elevated mean RHR as a continuous variable was an independent risk factor for mortality (hazard ratio [HR]: 3.69; 95\% confidence interval $[\mathrm{CI}]: 1.44-9.45 ; P=0.007)$ and invasive or non-invasive ventilator use (HR: 5.27; 95\% CI: 1.27-8.37; $P=0.014$ ). Mean RHR as tachycardia was also an independent risk factor for mortality (HR: 16.05; 95\% CI: 1.70-151.24; $P=$ 0.015 ) and ventilator use (HR: 14.45; 95\% CI: 1.42-147.23; $P=0.024)$ in the multivariable adjusted Cox proportional hazard regression model, as this relationship remained

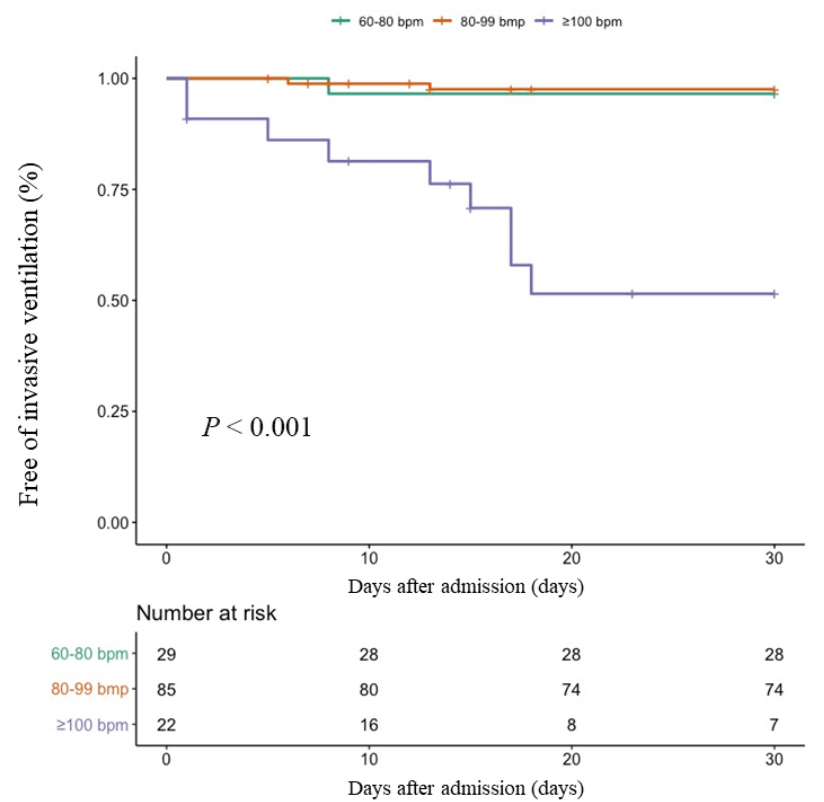

Figure 5: Kaplan-Meier estimates of patients not requiring invasive mechanical ventilation use by mean resting heart rate. bpm: beat per min.

statistically significant after adjusting for age, sex and other baseline parameters (Table 2).

The adjusted cubic spline model showed that elevated mean RHR as a continuous covariate was associated with higher risks of mortality (Figure 6A). The same spline model revealed a non-linear relationship between $\Delta \mathrm{HR}$ and mortality (Figure 6B). Increases and decreases in RHR were not significantly associated with a lower relative risk of death.

\section{DISCUSSION}

Our study revealed that the RHRs increased as the condition severity increased in COVID-19 patients, which improves predicting mortality compared with the knowledge of pulmonary function alone. However, the mechanism behind the increased RHR in severe COVID-19 patients and why it is positively correlated with a poor prognosis remains unclear.

RHR typically refers to the number of heartbeats per minute in a sober and quiet state, which is an important indicator of a patient's basic health condition. Except in patients with confirmed heart disease or shock, lower RHRs indicate better overall physical fitness, basal metabolism and heart function. An increased RHR is common in various pathological and non-pathological physical statuses and diseases such as tension, emotional agitation, fever, hypoxia, anaemia, systemic inflammation, hyperthyreosis, most acute and chronic cardiovascular diseases and metabolic syndrome. ${ }^{[10-13]}$ Because patients with SARS-CoV-19 


\begin{tabular}{|c|c|c|c|c|c|c|}
\hline Variables & Crude model & & Adjusted model I & & Adjusted model II & \\
\hline Mean RHR & HR (95\% Cl) & $P$-value & HR (95\% Cl) & $P$-value & HR $(95 \% \mathrm{Cl})$ & $P$-value \\
\hline \multicolumn{7}{|l|}{ Death } \\
\hline$<80$ & 1 (reference) & & 1 (reference) & & 1 (reference) & \\
\hline 80-99 bpm & $4.06(0.52-31.46)$ & 0.180 & $5.33(0.68-41.88)$ & 0.112 & $4.99(0.63-39.55)$ & 0.128 \\
\hline$\geq 100$ bpm & $24.83(3.26-189.20)$ & 0.002 & $26.36(3.39-204.85)$ & 0.002 & $16.05(1.70-151.24)$ & 0.015 \\
\hline Continuous variable & $5.73(2.85-11.50)$ & $<0.001$ & $5.02(2.47-10.20)$ & $<0.001$ & $3.69(1.44-9.45)$ & 0.007 \\
\hline \multicolumn{7}{|c|}{ Ventilator use } \\
\hline$<80$ & 1 (reference) & & 1 (reference) & & 1 (reference) & \\
\hline 80-99 bpm & $5.02(0.66-38.19)$ & 0.119 & $6.86(0.89-52.76)$ & 0.064 & $6.53(0.84-50.75)$ & 0.073 \\
\hline$\geq 100 \mathrm{bpm}$ & $37.53(4.97-283.36)$ & $<0.001$ & $57.90(7.26-461.52)$ & $<0.001$ & $14.45(1.42-147.23)$ & 0.024 \\
\hline Continuous variable & $7.08(3.71-13.52)$ & $<0.001$ & $8.14(3.88-17.06)$ & $<0.001$ & $3.27(1.27-8.37)$ & 0.014 \\
\hline
\end{tabular}

Adjusted model I: adjusted for sex and age; adjusted model II: adjusted for age, sex, NT-proBNP, Tnl, Hs-CRP, albumin, coronary artery disease, WBC, D-dimer, temperature for 3 days, $\beta$-blockers, creatinine, $\mathrm{SpO}_{2}$. We used the natural logarithms of the NT-proBNP and Tnl.

RHR: resting heart rate; $\mathrm{SpO}_{2}$ : pulse oxygen saturation; WBCs: white blood cells; Tnl: troponin I; NT-proBNP: N-terminal pro-B-type natriuretic peptide; HsCRP: hypersensitive C-reactive protein; HR: hazard ratio; $\mathrm{Cl}$ : confidence interval; bpm: beat per min.

A

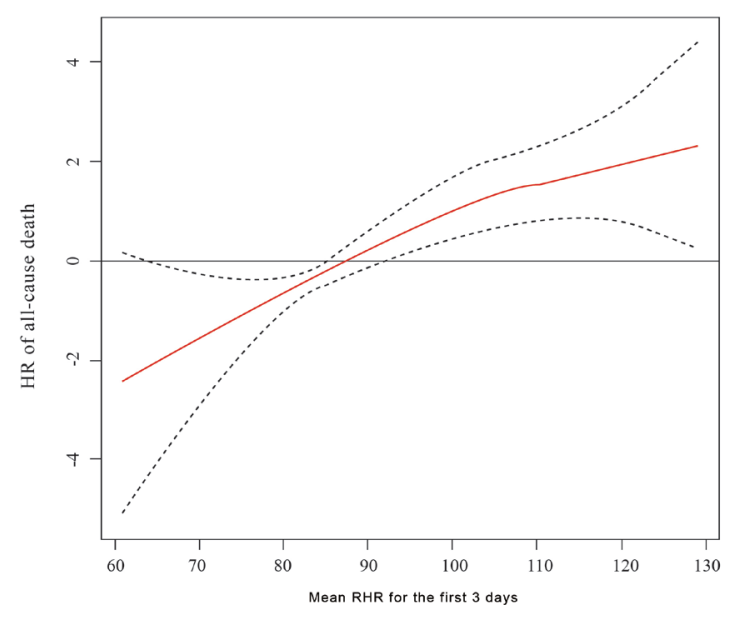

B

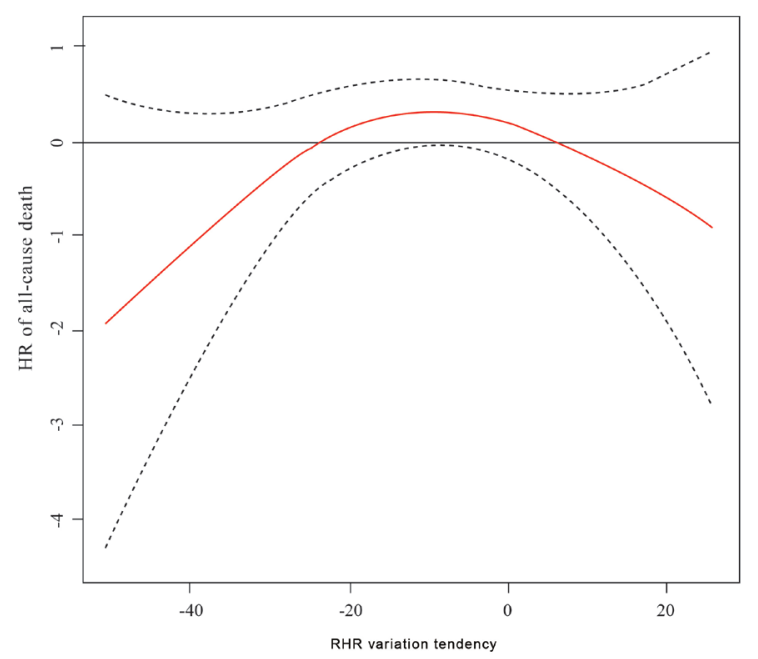

Figure 6: Relationships between mean resting heart rate and resting heart rate variation tendency and the risk of death. (A) The adjusted cubic spline model demonstrates the flexible relationship between mean RHR for the first 3 days and death. This curve (solid red) shows that a resting RHR $\geq 100$ beats/min was associated with an increasing trend in risk. The dashed black curves represent the upper and lower $95 \%$ confidence limits. The horizontal black line represents a hazard ratio of 1. (B) The adjusted cubic spline model demonstrates that the relationship between $\triangle R H R$ and mortality was non-linear. The dashed red curves represent the upper and lower $95 \%$ confidence limits. The horizontal black line represents a hazard ratio of 1 . Adjusted model II: adjusted for age, sex, NTproBNP, Tnl, Hs-CRP, albumin, coronary artery disease, WBC, D-dimer, temperature for 3 days, $\beta$-blocker, creatinine and $\mathrm{SpO}_{2}$.

RHR: resting heart rate; $\mathrm{SpO}_{2}$ : pulse oxygen saturation; WBC: white blood cells; Tnl: troponin I; NT-proBNP: N-terminal pro-B-type natriuretic peptide; Hs-CRP: hypersensitive C-reactive protein.

infections may exhibit nervousness and anxiety when admitted to the hospital and the heart rate at admission can be highly random (e.g., a patient was admitted to the hospital with an immediate heart rate of $120 \mathrm{bpm}$, which dropped to $62 \mathrm{bpm}$ on day 2), we used the average RHRs measured multiple times over the first 3 days after admission as the basis for classification. The baseline characteristics showed that as the RHRs increased, inflammatory factors, such as WBCs and hs-CRP, remained at higher levels, while albumin remained at a lower level, suggesting that systemic inflammatory cytokine storms and reduced nutrition in these patients may contribute to accelerating the RHRs with inflammatory stimuli and compensatory effects from the body. Although inflammatory cytokine mobilisation may also be attributed to glucocorticoid use, our guidelines for prescribing glucocorticoids are strict (e.g., confirmed acute respiratory distress syndrome diagnosed as per the Berlin definition, ${ }^{[14]}$ significantly increased procalcitonin with yellow sputum, confirmed pathogenic evidence and significantly decreased lymphocytes). Therefore, in 
most cases, the systemic inflammatory cytokine response already existed before the glucocorticoid use. Patients with higher D-dimers demonstrated increased RHRs, which coincided with previous study results on COVID-19. ${ }^{[15]}$ The mechanisms may include systemic inflammatory cytokine responses, which may lead to plaque rupture via local inflammation, haemodynamic changes and induction of procoagulant factors, which contribute to potential thrombosis. We also found that TnI, creatinine kinase MB isoenzyme and NT-proBNP were all significantly increased in patients with accelerated RHRs, inferring possible virus-induced cardiac damage. Huang et al. ${ }^{[16]}$ concluded that $19.7 \%$ of in-hospital COVID-19 patients $(82 / 416)$ experienced cardiac injury (defined as blood levels of hypersensitive $\operatorname{TnI}[\mathrm{hs}-\mathrm{TnI}]$ above the 99th percentile upper reference limit in this study). Cao et al. ${ }^{[15]}$ observed a similar definition and proportion $(17 \%, 21 / 145)$. In our database, the proportion reached $18.5 \%(25 / 136)$. Increased sympathetic tension caused by inflammation and hypoxia in COVID-19 patients can accelerate RHRs, exacerbate myocardial oxygen consumption and shorten coronary diastolic blood perfusion time, resulting in impaired endothelial function and aggravated atherosclerosis, leading to unstable plaque rupture, malignant arrhythmia and even death. Therefore, elevated myocardial injury markers are common under these conditions. ${ }^{[17]}$

Furthermore, men tended to have higher RHRs, which was also found in a similar study; $;^{[15]}$ thus, we assumed it was caused by the local overall male/female ratio. The higher proportion of antibiotic use before admission in patients with faster RHRs may be attributed to patients' earlier symptoms and increased awareness of autonomous medication, which also reflected the disease severity.

Elevated RHRs can increase morbidity and mortality rates for various diseases. Previous studies have shown that elevated RHRs may be strong predictors of cardiovascular death ${ }^{[3]}$ chronic obstructive pulmonary disease ${ }^{[18]}$ and metabolic syndrome. ${ }^{[11,12,19]}$ In addition to the chronic diseases, heart rate increases are also seen in patients with viral respiratory pathogen infections similar to COVID-19. In a study of severe acute respiratory syndrome (SARS) concerning 121 patients in 2006, $71.9 \%$ of the patients displayed tachycardia during the in-hospital care, but most of them developed into persistent type, including $40 \%$ with continued tachycardia during outpatient follow-up. Moreover, elevated heart rate in SARS was not associated with the risk of death. ${ }^{[20]}$ For other diseases like Middle East respiratory syndrome (MERS), only case reports showed sinus tachycardia or supraventricular tachycardia found in patients, ${ }^{[21,22]}$ and the predictive effect of RHRs on the prognosis has not been evaluated. In our study, Cox regression analysis suggested that an elevated mean RHR and RHR as tachycardia were independent risk factors for the all-cause mortality and ventilator use. These suggest that increased RHRs are neither clinical manifestations of patients' aggravated myocardial injuries nor the body's reaction to fever (our data showed that most patients maintained normal temperatures after admission). The RHRs tended to be a comprehensive manifestation of psychological stress, altered lung function, theoretic cardiovascular damage, hypoxia and cytokine storms in COVID-19 patients. Whether myocardial injury was an independent risk factor for adverse outcomes remains unknown. Huang et al. ${ }^{[16]}$ and Guo et al. ${ }^{[17]}$ suggested that myocardial injury was significantly associated with fatalities from COVID-19, whereas Zhu et al. ${ }^{[23]}$ found that concomitant cardiovascular manifestations were not independently associated with in-hospital adverse events in COVID-19 patients. Early studies showed that because angiotensin-converting enzyme 2 , the receptor for SARSCoV-2, was highly expressed on myocytes and vascular endothelial cells, which can induce viral attacks, direct cardiac involvement by the virus is possible. ${ }^{[24,25]}$ However, a more recent pathological study found scarce interstitial mononuclear inflammatory infiltrate in heart tissue without substantial myocardial damage in a patient with COVID-19, suggesting that COVID-19 might not directly impair the heart. ${ }^{[26]}$ Our results showed that elevated myocardial injury markers had no direct effect on the RHRs or the related prognosis; therefore, studies from other countries and larger populations are warranted to further confirm the outcomes of cardiac injury in COVID-19 patients.

Kaplan-Meier curves indicated that the in-hospital RHRs, especially RHRs as tachycardia, were directly related to poor prognosis among COVID-19 patients, emphasising the value of RHRs in early identification of severe and critical cases and early intervention strategies for critical patients' care and treatment.

In addition to the mean RHRs after admission, we also analysed the variation trend in the RHRs for 3 days, including RHRs showing both upward and downward trends. We failed to find a direct correlation between the variation tendency and patients' prognoses, possibly owing to the small sample size. However, negative outcomes from the RHR trends may suggest that the underlying absolute values of long-term RHRs are more meaningful than are the decreasing trends in COVID-19 patients over a short period after admission, indicating that heart rates are more reflective of long-standing health. This result was consistent with those of previous studies. ${ }^{[2,27]}$

Finally, some medications such as positive inotropic drugs and sedatives can interfere with RHRs. We did not count the use of positive inotropic drugs and sedatives 
in each patient, but we confirmed that all patients were free of these types of drugs in the first 3 days. A small proportion of patients in our study $(8 / 136)$ took drugs such as $\beta$-blockers, which can decrease RHRs. Although their taking the drugs neither affected analysis of the heart rate data nor affected better prognosis statistically significantly according to the baseline characteristics and adjusted model II in Cox regression analysis, our results suggest that drugs controlling RHRs may have potential therapeutic effect in COVID-19 patients.

\section{LIMITATIONS}

Our study had some limitations. First, owing to limited conditions, patients' heart rate data were measured multiple times for only the first 3 days. ECG monitoring and echocardiography examinations were unavailable for most patients after admission. We recorded the heart rate without accounting for the type of heart rhythm and changes in heart function, and the related cardiac complications and exact cause of death were difficult to be confirmed. Second, the study was a single-centre, retrospective study that included a small number of patients and was limited to severe and critical cases. Some patients remained under clinical observation and had not yet reached clinical endpoints; the $95 \%$ CI for RHRs $\geq 100 \mathrm{bpm}$ was wide as relatively fewer endpoints were met and the distribution had great disparity. These results should be corroborated by further prospective analysis. Third, although the study used the average data from the first 3 days after admission to represent patients' baseline RHRs, RHRs are highly variable indicators that depend on patients' physical condition and are susceptible to patients' emotional states. Moreover, in our study, evaluating the heart rate variability and responses to activities was impossible, although these measures are more meaningful and relevant indicators of endpoint events such as all-cause deaths. ${ }^{[28,29]}$

\section{Acknowledgement}

Ethics approval and consent to participate

The Ethics Committee of Peking University First Hospital approved this study (the ethics approval number is 2020-keyan-135), and all procedures and researches were in accordance with the 'Declaration of Helsinki'.

We thank Traci Raley, MS, ELS, from Liwen Bianji, Edanz Editing China (www.liwenbianji.cn/ac) for editing a draft of this manuscript.

\section{Source of Funding}

This study was supported by the Peking University First
Hospital Seed Foundation (No. 2020SF19).

\section{Conflict of Interests}

None declared.

\section{REFERENCES}

1. WHO COVID-19 Dashboard. Available at: https://covid19.who.int/. Accessed April 16, 2021.

2. Jouven X, Zureik M, Desnos M, Guérot C, Ducimetière P. Resting heart rate as a predictive risk factor for sudden death in middle-aged men. Cardiovasc Res 2001;50:373-8.

3. Cooney MT, Vartiainen E, Laatikainen T, Juolevi A, Dudina A, Graham IM. Elevated resting heart rate is an independent risk factor for cardiovascular disease in healthy men and women. Am Heart J 2010;159:612-9.e3.

4. Fox K, Ford I, Steg PG, Tendera M, Robertson M, Ferrari R. Heart rate as a prognostic risk factor in patients with coronary artery disease and left-ventricular systolic dysfunction (BEAUTIFUL): a subgroup analysis of a randomised controlled trial. Lancet 2008;372:817-21.

5. Guidance I. Clinical management of severe acute respiratory infection (SARI) when COVID-19 disease is suspected. Available at: https:// www.euro.who.int/en/health-topics/health-emergencies/coronaviruscovid-19/technical-guidance/2020/clinical-management-of-severeacute-respiratory-infection-when-novel-coronavirus-2019-ncovinfection-is-suspected-interim-guidance,-28-january-2020. Accessed June 21, 2020.

6. WMA - The World Medical Association-WMA Declaration of Helsinki - Ethical Principles for Medical Research Involving Human Subjects. Available at: https://www.wma.net/policies-post/wma-declaration-ofhelsinki-ethical-principles-for-medical-research-involving-humansubjects/. Accessed June 21, 2020.

7. Diagnosis and Treatment Protocol for Novel Coronavirus Pneumonia (Trial Version 5). Available at: http://www.gov.cn/zhengce/ zhengceku/2020-02/09/content_5476407.htm. Accessed June 21, 2020.

8. Diagnosis and Treatment Protocol for Novel Coronavirus Pneumonia (Trial Version 6). Available at: http://www.gov.cn/zhengce/ zhengceku/2020-02/19/content_5480948.htm. Accessed June 21, 2020.

9. Diagnosis and Treatment Protocol for Novel Coronavirus Pneumonia (Trial Version 7). Available at: http://busan.china-consulate.org/chn/zt/4/ t1754009.htm. Accessed June 21, 2020.

10. Qiu S, Cai X, Sun Z, Li L, Zuegel M, Steinacker JM, et al. Heart Rate Recovery and Risk of Cardiovascular Events and All-Cause Mortality: A Meta-Analysis of Prospective Cohort Studies. J Am Heart Assoc [Internet]. 2017; 6(5):e005505.

11. Shapira I, Rogowski O, Berliner S. Abstract: P754 Resting Heart Rate Is Associated With The Metabolic Syndrome - An Additional Connection Between Resting Heart Rate And Cardiovascular Disease [Internet]. Vol. 10, Atherosclerosis Supplements 2009;10:p. e118.

12. Liu X, Luo X, Liu Y, Sun X, Han C, Zhang L, et al. Resting heart rate and risk of metabolic syndrome in adults: a dose-response meta-analysis of observational studies. Acta Diabetol 2017;54:223-35.

13. Fox K, Bousser M-G, Amarenco P, Chamorro A, Fisher M, Ford I, et al. Heart rate is a prognostic risk factor for myocardial infarction: a post hoc analysis in the PERFORM (Prevention of cerebrovascular and cardiovascular Events of ischemic origin with teRutroban in patients with a history oF ischemic strOke or tRansient ischeMic attack) study population. Int J Cardiol 2013;168:3500-5.

14. Redant S, Devriendt J, Botta I, Attou R, De Bels D, Honoré PM, et al. Diagnosing Acute Respiratory Distress Syndrome with the Berlin Definition: Which Technical Investigations Should be the Best to Confirm it? J Transl Int Med 2019;7:1-2.

15. Zhou F, Yu T, Du R, Fan G, Liu Y, Liu Z, et al. Clinical course and risk 
factors for mortality of adult inpatients with COVID-19 in Wuhan, China: a retrospective cohort study. Lancet 2020;395:1054-62.

16. Shi S, Qin M, Shen B, Cai Y, Liu T, Yang F, et al. Association of Cardiac Injury With Mortality in Hospitalized Patients With COVID-19 in Wuhan, China. JAMA Cardiol 2020;5:802-10.

17. Guo T, Fan Y, Chen M, Wu X, Zhang L, He T, et al. Cardiovascular Implications of Fatal Outcomes of Patients With Coronavirus Disease 2019 (COVID-19). JAMA Cardiol 2020; 5:811-8.

18. Jensen MT, Marott JL, Lange P, Vestbo J, Schnohr P, Nielsen OW, et al. Resting heart rate is a predictor of mortality in COPD. Eur Respir J 2013;42:341-9.

19. Wu X, Du R, Hu C, Cheng D, Ma L, Li M, et al. Resting heart rate is associated with metabolic syndrome and predicted 10-year risk of cardiovascular disease: a cross-sectional study. J Diabetes 2019;11:88494. .

20. Yu CM, Wong RSM, Wu EB, Kong SL, Wong J, Yip GW-K, et al. Cardiovascular complications of severe acute respiratory syndrome. Postgrad Med J 2006;82:140-4.

21. Al-Abdallat MM, Payne DC, Alqasrawi S, Rha B, Tohme RA, Abedi GR, et al. Hospital-associated outbreak of Middle East respiratory syndrome coronavirus: a serologic, epidemiologic, and clinical description. Clin Infect Dis 2014;59:1225-33.

22. Saad M, Omrani AS, Baig K, Bahloul A, Elzein F, Matin MA, et al. Clinical aspects and outcomes of 70 patients with Middle East respiratory syndrome coronavirus infection: a single-center experience in Saudi Arabia. Int J Infect Dis 2014;29:301-6.

23. Liu R, Ming X, Xu O, Zhou J, Peng H, Xiang N, et al. Association of Cardiovascular Manifestations with In-hospital Outcomes in Patients with COVID-19: A Hospital Staff Data. Am Heart J 2020;226:174-87

24. Chen L, Li X, Chen M, Feng Y, Xiong C. The ACE2 expression in human heart indicates new potential mechanism of heart injury among patients infected with SARS-CoV-2. Cardiovasc Res 2020;116:1097-100.

25. Gallagher PE, Ferrario CM, Tallant EA. Regulation of ACE2 in cardiac myocytes and fibroblasts. Am J Physiol Heart Circ Physiol 2008;295:H2373-9.

26. Xu Z, Shi L, Wang Y, Zhang J, Huang L, Zhang C, et al. Pathological findings of COVID-19 associated with acute respiratory distress syndrome. Lancet Respir Med 2020;8:420-2.

27. Böhm M, Swedberg K, Komajda M, Borer JS, Ford I, Dubost-Brama A, et al. Heart rate as a risk factor in chronic heart failure (SHIFT): the association between heart rate and outcomes in a randomised placebocontrolled trial. Lancet 2010;376:886-94.

28. Chang WL, Lee JT, Li CR, Davis AHT, Yang CC, Chen YJ. Effects of Heart Rate Variability Biofeedback in Patients With Acute Ischemic Stroke: A Randomized Controlled Trial. Biol Res Nurs 2020;22:34-44.

29. Chang YM, Huang YT, Chen IL, Yang CL, Leu SC, Su HL, et al. Heart rate variability as an independent predictor for 8-year mortality among chronic hemodialysis patients. Sci Rep 2020;10:881.
How to cite this article: Jin $\mathrm{H}$, Yang $\mathrm{S}$, Yang $\mathrm{F}$, Zhang $\mathrm{L}$, Weng $\mathrm{H}$, Liu $S$, et al. Elevated resting heart rates are a risk factor for mortality among patients with coronavirus disease 2019 in Wuhan, China. J Transl Intern Med 2021; 9: 285-93. 